\section{Whole-Body FDG PET Imaging in Oncology: Clinical Reports}

\author{
P.F. Rambaldi
}

New York, NY: Springer, 2014, 352 pages, $\$ 99$

By presenting a picture of human anatomy on which biologic information is added, PET/CT allows better delineation of disease within or between structures, as well as guiding surgical and radiation planning and biopsy. It also allows prognostic stratification that has immediate and sometimes dramatic consequences, not only on the subsequent choice of treatment but also on the quality of life of patients and their families. This atlas or manual of PET/CT provides an educational approach toward defining the principles and good practices of PET/CT and thus is essential for practitioners of nuclear medicine and radiology who want to learn more about molecular diagnostics and therapeutics in cancer management.

The 80 chapters are a large collection of clinical cases in oncology with accompanying whole-body ${ }^{18} \mathrm{~F}-\mathrm{FDG}$ PET/CT images. The 12 contributors of the cases are mostly from Naples, Italy. The aim of the book is to promote an integrated approach to the use of PET/CT, and detailed attention is therefore paid to the clinical history and diagnostic question. The 12 parts of this book are broadly structured according to anatomic region and cover a wide range of common diseases likely to be imaged using PET/CT, including those of the biliary system, head and neck, colon-rectum, esophagus, lymphatic system, breast, pancreas, lung, stomach, and urinary tract, as well as gynecologic diseases and melanoma. The focus of every case is to provide guidance on the clinical report, which is the official tool for communicating with both the referring physician and the patient. For this reason, the report needs to be clear, understandable, and written in a shared language. The advice on report preparation is strongly supported by informative PET, CT, and PET/CT images of each disease. The illustrative images are clear and informative. There are no tables or references.

This book should be valuable to all those training or working in the field of nuclear medicine, radiology, and oncology who want to ensure that they are best placed to contextualize, interpret, and report the findings of PET/CT, which can have such a dramatic impact on prognosis, therapeutic response, and quality of life.

\section{E. Edmund Kim \\ University of California at Irvine 101 The City Dr. S. Orange, CA 92868 E-mail: edmundek@uci.edu}

\section{Core Radiology: A Visual Approach to Diagnostic Imaging}

J. Mandell, ed.

New York, NY: Cambridge University Press, 2013, 887 pages, $\$ 117.40$

Diagnostic imaging is becoming exceedingly complex. As the field adds a new imaging technique or improves with computerbased digital technique, the analysis and correct differential diagnosis often present a dilemma for physicians, who will have considerable difficulty in learning everything they need to master.

Core Radiology: A Visual Approach to Diagnostic Imaging, written by 36 experienced contributors, is a comprehensive, up-to-date resource for learning or reviewing diagnostic images. It has 12 chapters organized by organ system, including thoracic, gastrointestinal, genitourinary, neurologic, musculoskeletal, breast, cardiovascular, and pediatric topics, as well ultrasound imaging. The chapter on nuclear imaging deals with PET/CT and cardiac studies. The last chapter handles the physics of imaging and includes contrast medium and reaction. The clearly formatted design integrates more than 1,200 superb clinical images with 300 color illustrations, and there is concise bulleted text to facilitate efficient and streamlined learning. All subjects covered by America Board of Radiology core examination are included.

With clinical cases related to multiple clinical entities presented in depth, this book is designed to be a teaching manual and everyday companion for residents training in radiology or nuclear medicine. The book also helps busy practitioners determine the pathologic cause of a finding on various diagnostic images. I highly recommend this essential book to trainees in the field of diagnostic imaging.

\author{
E. Edmund Kim \\ University of California at Irvine \\ 101 The City Dr. S. \\ Orange, CA 92868 \\ E-mail: edmundek@uci.edu
}

DOI: 10.2967/jnumed.114.146340
DOI: 10.2967/jnumed.114.148700

COPYRIGHT (c) 2014 by the Society of Nuclear Medicine and Molecular Imaging, Inc. 